\title{
DETECTION OF THE IRRADIATED RED DWARF IN WY SGE (NOVA 1783)
}

\author{
M. W. SOMERS ${ }^{1}$, K. MUKAI ${ }^{2}$, T. NAYLOR ${ }^{1}$, F. A. RINGWALD ${ }^{1}$ \\ 1. Department of Physics, Keele University, ST5 5BG, UK \\ 2. Office for Guest Investigator Programs, Code 668, NASA/ \\ Goddard Space Flight Center, Greenbelt, MD 20771, USA
}

\begin{abstract}
We present infrared (IR) photometry and optical spectroscopy of the eclipsing old nova WY Sge. According to hibernation theorists the disc in a system a few centuries after outburst should be significantly fainter than in other, more recent, old novae but similar to the discs of dwarf novae in quiescence. Despite the apparent faintness of the late type star we have sufficient information to infer that the face closest to the white dwarf (WD) is irradiated.
\end{abstract}

\section{Introduction}

WY Sge is an object of considerable interest because it is the oldest securely recovered nova, and thus may help our understanding of the evolution of classical nova systems between outbursts. Shara et al. (1986) suggest that a few hundred years after outburst old novae should fade considerably. Naylor et al. (1992) have argued against such a model. The IR photometry (see also Somers et al. 1996), and optical spectroscopy presented here, show that WY Sge is not in hibernation.

\section{Results}

\subsection{INFRARED PHOTOMETRY}

The flux remaining in eclipse provides an upper limit to the contribution of the late type star. It implies that the late type star in WY Sge provides less than $50 \%$ of the flux in the $K$-band. Comparison with dwarf novae (Szkody \& Mateo 1986) shows that all have the secondary star contributing around $63 \%$ or more of the flux at $K$ (assuming all the disc light is eclipsed). If we limit ourselves to systems with a period greater than $3 \mathrm{~h}$ (and thus a 
similarly sized secondary to WY Sge) the secondary star provides $\gtrsim 76 \%$ of the light in all cases, with $\gtrsim 91 \%$ for the two eclipsing systems (U Gem, IP Peg). The fraction of light from the late type star in the non-eclipsing nova-like variable IX Vel (Haug 1988) in the $K$-band is about $20 \ldots 40 \%$, in agreement with WY Sge.

At $K$ maximum light occurs at phase $0.32 \pm 0.01$ which implies that the out of eclipse modulation is not purely ellipsoidal. We have modelled this in terms of a gravity and limb darkened Roche lobe being irradiated by a source at the position of the WD and shaded by the disc.

\subsection{SPECTROSCOPY}

The spectra taken in the red arm of ISIS on the WHT were shifted and added on the $K_{2}$ velocity derived from the narrow line emission. Several absorption features are apparent, most noticeably the TiO bands. We use the flux density deficit method in the TiO bands (Wade \& Horne 1988) to calculate the fraction of light originating from the late type star and its spectral type. The secondary is of spectral type M1-M5, with a best estimate of M4. The ISIS blue arm spectra covered the region around He II and $\mathrm{H} \beta$. In the individual spectra it is apparent that there is a sharp component of the $\mathrm{H} \beta$ profile that moves with phase. The narrow component is phased correctly to be tracing the velocity of the late type star; it is anti-phased with the inner disc, which roughly coincides with the velocity of the WD. Narrow line emission is indicative of irradiation of the inner face of the late type star.

\section{Conclusions}

We show that, contrary to the predictions of hibernation theory, WY Sge appears to have a brighter disc, at both IR and optical wavelengths, than other similar period and inclination dwarf novae. The disc is of comparable brightness as that in the nova-like variable IX Vel.

We also find that the secondary is irradiated on the face closest to the WD. The origin of the irradiation is unclear; it is either the remnant heating from the cooling WD after the nova explosion or accretion irradiation.

\section{References}

Haug, K., 1988, MNRAS, 235, 1385

Naylor, T., Charles, P. A., Mukai, K., Evans, A., 1992, MNRAS, 258, 449

Shara, M. M., Livio, M., Moffat A. F. J., Orio, M., 1986, Ap. J., 311, 163

Somers, M. W., Mukai, K., Naylor, T., 1996, MNRAS, in preparation

Szkody, P., Mateo, M., 1986, AJ, 92, 483

Wade, R. A., Horne, K., 1988, Ap. J., 324, 411 